\title{
Efficacy and safety of probiotics as adjuvant agents for Helicobacter pylori infection: A meta-analysis
}

\author{
ZHIFA LV $^{1 *}$, BEN WANG $^{1^{*}}$, XIAOJIANG ZHOU ${ }^{1 *}$, FUCAI WANG $^{1}$, \\ YONG XIE $^{1}$, HUILIE ZHENG ${ }^{2}$ and NONGHUA LV ${ }^{1}$ \\ ${ }^{1}$ Department of Gastroenterology, The First Affiliated Hospital of Nanchang University; \\ ${ }^{2}$ School of Public Health, Nanchang University, Nanchang, Jiangxi, P.R. China
}

Received April 29, 2014; Accepted December 5, 2014

DOI: $10.3892 / \mathrm{etm} .2015 .2174$

\begin{abstract}
The aim of the present study was to determine whether probiotics could help to improve the eradication rates and reduce the side effects associated with anti-Helicobacter pylori treatment, and to investigate the optimal time and duration of probiotic administration during the treatment, thus providing clinical practice guidelines for eradication success worldwide. By searching Pubmed, Embase, the Cochrane Central Register of Controlled Trials and the Science Citation Index, all the randomized controlled trials (RCTs) comparing probiotics as adjuvant agents of anti-H.pylori standard triple-therapy regimens with placebo or no treatment were selected. Statistical analysis was performed with the Comprehensive Meta Analysis Software. Subgroup, meta-regression and sensitivity analyses were also carried out. Twenty-one RCTs involving a total of 3,814 participants met the inclusion criteria. The pooled eradication rates of the probiotic group were $80.3 \%(1,709 / 2,128)$ by intention-to-treat (ITT) and $83.8 \%(1,709 / 2,039)$ by pro-protocol analyses; the pooled relative risk (RR) by ITT for probiotic supplementation versus treatment without probiotics was 1.12 [95\% confidence interval (CI), 1.06-1.19]. A reduced risk of overall H. pylori therapy-related adverse effects was also found with probiotic supplementation (RR, 0.60; 95\% CI, 0.40-0.91). The subgroup analyses showed that probiotic supplementation prior and subsequent to the treatment regimen both improved eradica-
\end{abstract}

Correspondence to: Dr Yong Xie, Department of Gastroenterology, The First Affiliated Hospital of Nanchang University, 17 Yongwaizheng Street, Nanchang, Jiangxi 330000, P.R. China

E-mail: xieyong_med@163.com

Dr Huile Zheng, School of Public Health, Nanchang University, 461 Bayi Street, Nanchang, Jiangxi 330006, P.R. China

E-mail: zhenghuilie@163.com

${ }^{*}$ Contributed equally

Key words: probiotics, Helicobacter pylori, adjuvant treatment, meta-analysis tion rates for H. pylori infection. Furthermore, probiotic treatment lasting $>2$ weeks and including Lactobacillus or multiple probiotic strains significantly enhanced the efficacy. In conclusion, supplementation with probiotics for $H$. pylori eradication may be effective in increasing eradication rates and decreasing therapy-related side effects. Probiotic administration prior or subsequent to therapy and for a duration of $>2$ weeks may increase the eradication efficacy.

\section{Introduction}

Helicobacter pylori is a Gram-negative spiral bacterium that colonizes the gastric mucosa. H. pylori infection affects $70-90 \%$ the population in developing countries, and $25-50 \%$ of the population in developed countries (1). Standard triple-therapy regimens with a proton-pump inhibitor (PPI) and two of amoxicillin, clarithromycin and nitroimidazole have been used for the eradication of $H$. pylori; however, their efficacy has been declining with the increasing resistance of $H$. pylori to antibiotics, and the $H$. pylori eradication failure rate varies widely, from 10 to $45 \%$ (2). At present, the administration of antibiotics for 10-14 days or high-dose PPI (twice a day) has been recommended for $H$. pylori eradication therapy by the Maastricht IV consensus conference (3); this has resulted in the increased incidence of undesirable side effects, such as antibiotic-associated diarrhea, nausea or vomiting, during anti- $H$. pylori therapy, which can lead to reduced compliance (4). Among the alternative anti-H. pylori options that have been considered, probiotics have attracted substantial interest. Previous studies have shown that probiotics, predominantly including Lactobacillus, Saccharomyces boulardii and Bifidobacterium, demonstrate anti-H. pylori activity in vitro and in animal models of $H$. pylori infection (5-8). Probiotics have also been used as an adjuvant therapy to $H$. pylori infection in order to reduce the side effects of antibiotics and improve the eradication rates (9-11); however, the results have been inconsistent, with certain studies showing that adjuvant probiotics did not improve eradication rates or reduce the side effects (12-14).

Previous meta-analyses have demonstrated that probiotics, as adjuvant agents, have a positive effect on improving eradication rates and reducing adverse events (15-20); however, certain recent studies have produced results that are inconsistent with 
those of the previous meta-analyses $(21,22)$. Furthermore, the appropriate timing and duration of probiotic administration are indeterminate $(23,24)$. Miscellaneous probiotics may be used in an anti-H. pylori treatment regimen, but it is unclear whether the efficacy of different probiotics is similar. As such, an updated meta-analysis of randomized controlled trials (RCTs) comparing the eradication rates and adverse events of probiotics as an adjuvant treatment with those of a placebo (or blank control) in participants with $H$. pylori infection is required. The aim of the present study was to evaluate, by meta-analysis, the efficacy and safety of the administration of probiotics as adjuvant agents of standard triple-therapy regimens for $H$. pylori infection, and to investigate the appropriate timing and duration of the probiotic administration in order to provide evidence to support this use of probiotics in clinical practice.

\section{Materials and methods}

Study sources and search methods. The present meta-analysis was developed according to the Preferred Reporting Items for Systematic Reviews and Meta-Analyses statement guidelines (25). Pubmed (1966 to November 2013), Embase (1946 to November 2013), the Cochrane Central Register of Controlled Trials (Issue 11, 2013) and the Science Citation Index (SCI; 1945 to November 2013) were searched according to Medical Subject Heading and text terms: (Helicobacter pylori OR H. pylori) AND (probiotic OR probiotics OR yeast OR yeasts OR yogurt OR Lactobacillus OR Bifidobacterium OR Saccharomyces). Authors were also asked to provide unpublished randomized trial results. In addition, the ClinicalTrials.gov website (https://clinicaltrials.gov/) was searched for registered RCTs whose results had not yet been published, and relevant studies were identified from the references.

Inclusion and exclusion criteria. Articles that were eligible for inclusion in the meta-analysis met the following inclusion criteria: i) RCTs; ii) any age, endoscopic findings and symptoms at the time of enrollment; iii) confirmation of eradication outcome by urea breath test, histology or H. pylori stool antigen $\geq 4$ weeks after therapy; iv) trials comparing at least two branches of treatment consisting of a control group (with placebo or no additional intervention) and an experimental group (the standard triple-therapy regimen plus probiotics); v) restriction of the species of probiotics to Lactobacillus, Bifidobacterium, Saccharomyces or a mixture of the three; vi) obtainable eradication rates.

The exclusion criteria for the meta-analysis were as follows: i) Undeterminable eradication rates; ii) use of agents other than probiotics as the adjuvant therapy for $H$. pylori infection in the experimental group; iii) articles without full-text; iv) studies in languages other than English.

Validity assessment. Two reviewers independently, but not blinded to the authors or journal, assessed the quality of the studies that met the inclusion criteria. Any disagreements between the reviewers were resolved by consulting a third reviewer. The quality of the studies was assessed by the Jadad scale $(26,27)$. The scores, from 0 to 5 , were evaluated according to three criteria: Randomization, double blinding and description of withdrawals and dropouts $(26,27)$. To avoid the duplication of data, if trials were published repeatedly by the same authors or institutions, only the most recently published article was included.

Data extraction. Standardized data abstraction sheets were prepared. Data were extracted for study quality and type; the timing of probiotic administration; duration of eradication treatment; duration of probiotic treatment; species of probiotics; location of trials; time of publication; anti-H. pylori regimens; number and age of enrolled patients; diagnostic methods for detecting $H$. pylori infection prior to enrollment and subsequent to study completion; eradication rates by intention-to-treat (ITT) analysis; rates of successful and failed eradication; and total side effects (diarrhea, vomiting nausea, taste disturbance, epigastric pain and total adverse effects) from all included studies.

Statistical analysis. Statistical analysis was performed with the Comprehensive Meta-Analysis Software (version 2; Biostat, Inc., Englewood, NJ, USA). The primary outcomes for the meta-analysis were the $H$. pylori eradication rates and the side effects among the trials comparing probiotic and control arms, based on ITT and pro-protocol (PP) analysis. The efficacy of H. pylori eradication was measured using relative risk (RR) to compare the frequency of eradication in the probiotic arm with that in the control arm.

The RRs for all studies were pooled into a summary RR, using either a fixed- or random-effects model, based on inverse variance methods. If the heterogeneity had a statistically significant difference, the random-effects model was employed; if not, the fixed-effects model was adopted. P-values and 95\% confidence intervals (CIs) were provided for the summary RRs. The heterogeneity index $\left(\mathrm{I}^{2}\right)$ was additionally calculated. Other assessments of heterogeneity were accomplished using the Q-test, and a Z-test was employed to assess the pooled effects. Funnel plots, Egger's test and Begg's test were utilized to estimate the publication bias. Meta-regression analyses were performed to interpret the reasons for the heterogeneity.

Subgroup analysis. Subgroup analysis for the meta-analysis was performed depending on the time that the probiotics were administered ['before' (used prior to the eradication regimens), 'same' (simultaneously with the eradication regimens) and 'after' (beginning with the eradication regimens and continuing subsequent to the eradication regimens)], the regimens utilized, the duration of the probiotic treatment ( $\leq 2$ weeks and $>2$ weeks), the species of probiotics, the age of the subjects, the Jadad score $(>2$, and $\leq 2)$, the PPIs of the experimental group and the duration of the eradication regimens.

\section{Results}

Description of the studies. The bibliographical search yielded a total of 2,653 studies. Among the studies from Pubmed, the Cochrane Central Register of Controlled Trials, Embase and the SCI, another 2,478 articles were excluded subsequent to examining the article type. Having excluded any duplicates, 


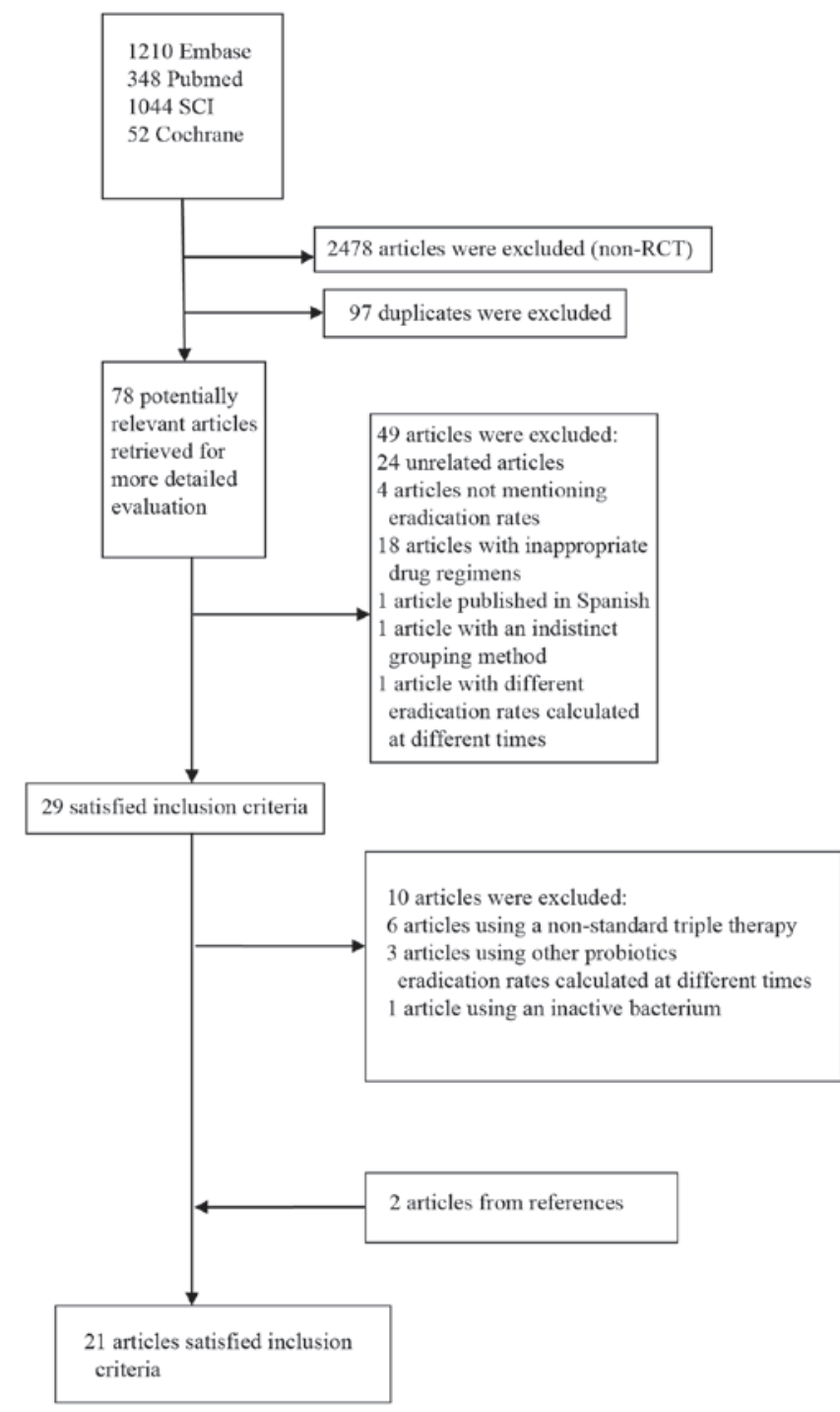

Figure 1. Flow diagram of the trials identified and selected. SCI, Science Citation Index; RCT, randomized controlled trial.

78 potentially relevant articles were retrieved for more detailed assessment. Following examinations of the title and abstract, another 24 unrelated articles, four articles that did not mention eradication rates, 18 articles with inappropriate drug regimens and one study published in Spanish (28) were excluded. The full-text articles were then reviewed and another two articles were excluded, one of which was excluded for indistinct grouping methods (29) and the other as a result of eradication rates being calculated at different times. Twenty-nine articles were further evaluated for details. Six articles were excluded due to a non-standard triple therapy regimen $(9,12,30-33)$, three articles were excluded due to Lactobacillus, Bifidobacterium or Saccharomyces not being used in the eradication regimen (34-36) and one article due to an inactive bacterium being used (37). Two articles from the studied references were additionally included in the meta-analysis $(38,39)$. Twenty-one RCTs ultimately met the inclusion criteria (11,38-57) (Table I) (Fig. 1).

Two institutions published two similar articles $(40,41)$, in Italy. This triggered a concern about duplication of data; however, following a careful review of the articles in question, it was decided that the two articles were separate trials.
Efficacy of $H$. pylori eradication. The 21 RCTs included 3,814 patients in total, of whom 21 patients were in the probiotic group and 1,529 in the control group. The pooled eradication rate of the probiotic group was $80.3 \%(1,709 / 2,128)$ by intention-to-treat (ITT) and $83.8 \%(1,709 / 2,039)$ by PP analysis; the eradication rate in the probiotic group was higher than that in the control group (80.3 vs. $72.2 \%)$ with a statistically significant difference $(\mathrm{Z}=3.917, \mathrm{P}<0.001)$. Using the random-effects model, the values of $\mathrm{I}^{2}=52.3 \%$ and $\mathrm{P}=0.003$ were obtained. The RR from a pooled analysis of the selected studies was 1.12 (95\% CI, 1.06-1.19) by ITT analysis (Fig. 2).

Subgroup analyses. Multiple subgroup analyses were carried out to explain the heterogeneity by stratifying the studies based on the timing of probiotic supplementation ('before', 'same' and 'after'), eradication regimens, duration of probiotic supplementation, species of probiotics, age of patients (adults and children) and Jadad scores.

Therewere 11 trials in which the probiotics wereadministered subsequent to the eradication regimens $(38,40-46,50,52,56)$. The pooled analysis showed that the RR was 1.15 (95\% CI, 1.10-1.21) according to the random-effects model. The RR of the eight studies in which probiotics were used simultaneously with the eradication regimens was 1.04 (95\% CI, 0.92-1.19) by the random-effects mode $(11,51,39,47,48,53,54,57)$. The RR for the probiotics used prior to the eradication regimens in the four RCTs was 1.21 (95\% CI, 1.10-1.32) by the random-effects model $(39,49,55,56)$. When the probiotics were administered prior or subsequent to the standard triple therapy, the differences between the experimental and control groups were statistically significant. When probiotics were used concurrently with the eradication regimens, no significant difference was found between the experimental group and the control group. This demonstrated that the timing of probiotic supplementation, i.e. prior or subsequent to the standard triple-therapy regimen, could improve the eradication rate (Fig. 3).

Subgroup analyses were additionally performed according to the eradication regimens (PPI plus amoxicillin and clarithromycin, PPI plus clarithromycin and tinidazole). The RRs analyzed in the random-effects model were 1.14 (95\% CI, 1.07-1.21) and 1.04 (95\% CI, 0.92-1.19) for the two regimens, respectively. The combination of the standard triple-therapy regimen (PPI, amoxicillin and clarithromycin) with probiotics achieved a significantly higher eradication rate than that obtained without probiotics; however, the combination of the PPI plus clarithromycin and tinidazole triple therapy with probiotics did not significantly improve the eradication rate for H. pylori.

According to the subgroup analyses of the duration of probiotic supplementation, the RR for durations of $\leq 2$ weeks was 1.04 (95\% CI, 0.98-1.11) by random-effects model, and the RR for durations of $>2$ weeks was 1.17 (95\% CI, 1.12-1.23) by random-effects model. The results showed that probiotic supplementation for $>2$ weeks could improve the eradication of $H$. pylori.

According to subgroup analyses of species, the RR for Lactobacillus was 1.14 (95\% CI, 1.08-1.25), the RR of Saccharomyces boulardii was 1.06 (95\% CI, 0.89-1.23) and that of Bifidobacterium was 1.25 (95\% CI, 0.86-1.82) (all by random-effects model). The RR in the multiple strains 


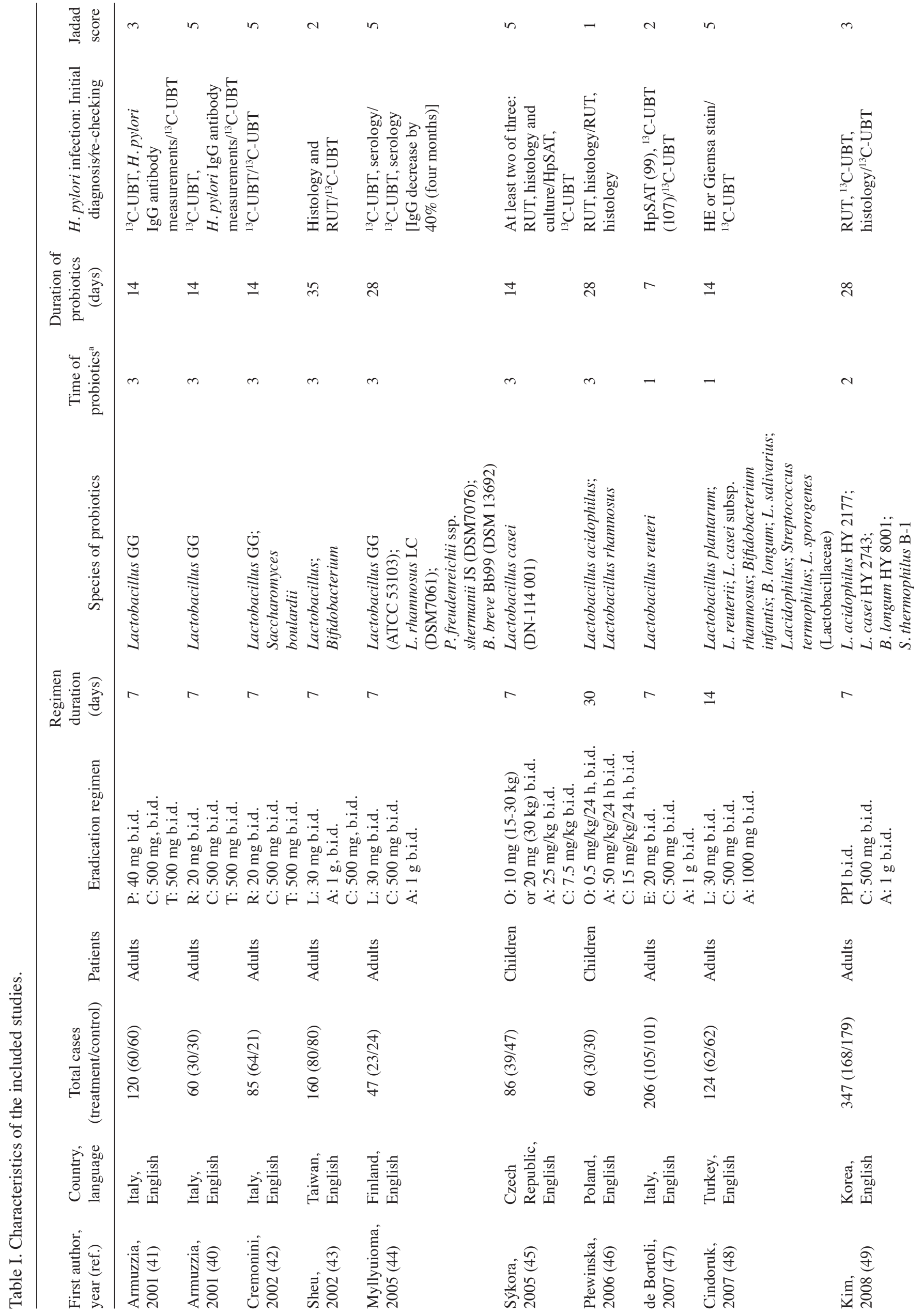




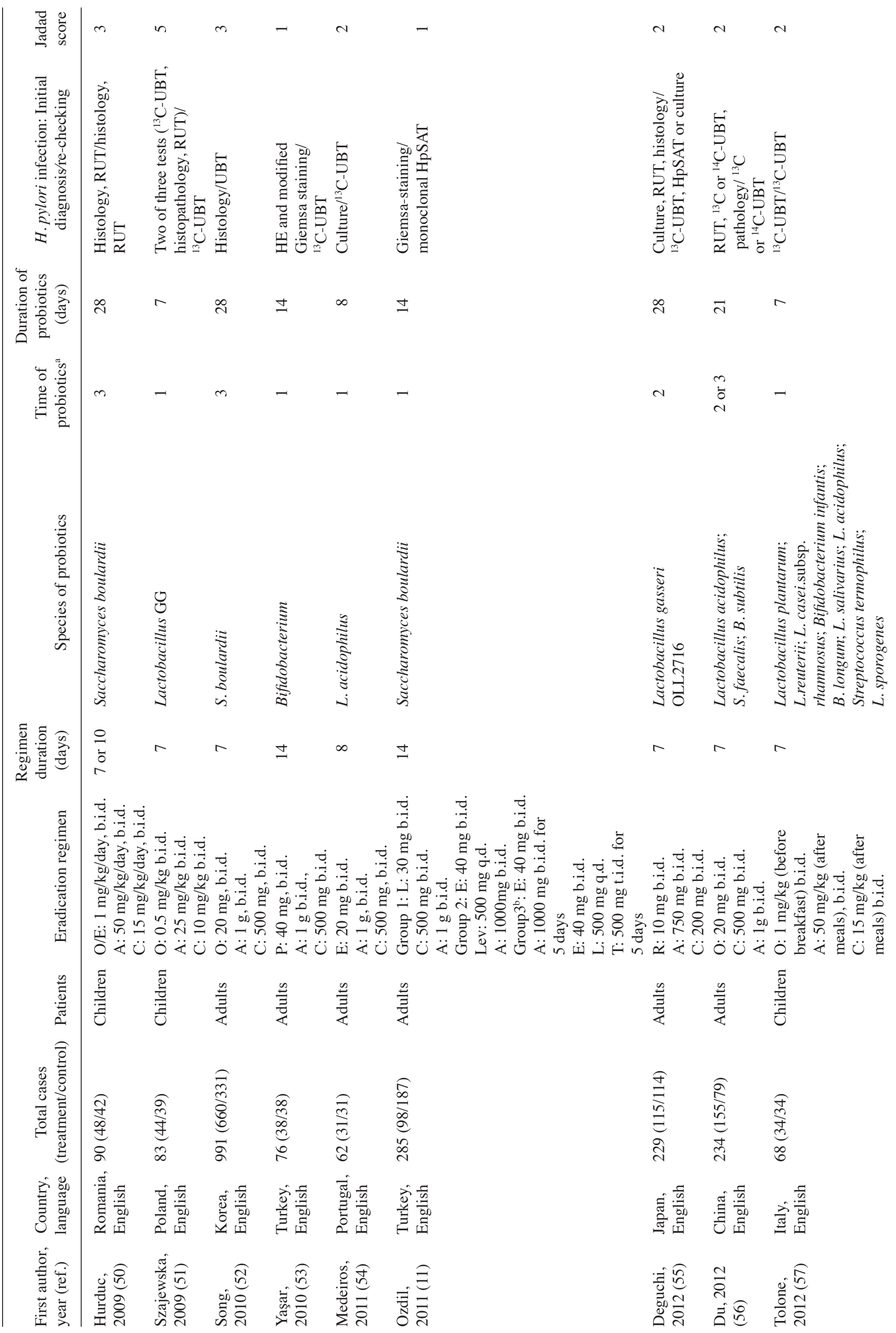


subgroup was 1.15 (95\% CI, 1.08-1.22). The findings demonstrated that the use of Lactobacillus and multiple probiotic strains as adjuvant agents could improve the effectiveness of the $H$. pylori eradication to a greater extent than the control treatment; however, the administration of Bifidobacterium or Saccharomyces boulardii did not appear to improve eradication during anti- $H$. pylori treatment.

In the adult and children subgroup analyses, the RRs were 1.08 (95\% CI, 1.01-1.16) and 1.22 (95\% CI, 1.11-1.34), respectively, by the random-effects model. The results showed the enhanced efficacy of probiotic supplementation relative to that of the control treatment $(\mathrm{P}<0.001)$ in both adults and children.

There were 11 trials in which the Jadad scores were $\geq 3$, indicating that their quality was high $(40-42,44,45,48-52,56)$. The Jadad scores were $<3$ in 10 studies, which indicated that their quality was low $(11,30,38,39,42,44-53,56,57)$. According to the pooled analysis of the high-quality trials, the summary RR was 1.13 (95\% CI, 1.08-1.19) by random-effects model. The RR of the studies of low quality was 1.12 (95\% CI, 1.00-1.25) by random-effects model. The high-quality studies all showed the benefit of probiotic supplementation compared with the control treatment $(\mathrm{P}<0.001)$, but no significant difference was found between the treatments in the low-quality studies $(\mathrm{P}=0.053)$.

Subgroup analyses were also performed based on the different PPIs of the trial groups and the duration of the eradication regimens. In the omeprazole and rabeprazole subgroups, the differences between the probiotic and control groups were statistically significant $(\mathrm{P}<0.001$ and $\mathrm{P}=0.034$, respectively). Significant differences were also found for eradication durations of seven and 10 days $(\mathrm{P}<0.001$ and $\mathrm{P}=0.012$, respectively).

Adverse events. A total of 16 out of the 21 trials described side effects, including diarrhea, vomiting and nausea, and epigastric pain $(30,38,39,42,44-53,56,57)$. Ten RCTs had data on total side effects $(38,42,44,45,47,49,50,52,53,56)$. The summary RR was 0.60 (95\% CI, 0.40-0.91) according to random-effects model analysis $\left(\mathrm{I}^{2}=83.72 \%, \mathrm{P}<0.001\right)$ (Fig. 4). Twelve RCTs reported the data for diarrhea $(30,38,39,42,44$, $46-49,51,52,57), 10$ for vomiting and nausea $(30,38,42,44,46$ $-49,52,57)$ and eight for epigastric pain $(42,44,46-49,52,57)$. The pooled RRs were 0.42 for diarrhea (95\% CI, 0.24-0.73) and 0.56 for vomiting and nausea (95\% CI, 0.27-1.16) by random-effects model $\left(\mathrm{I}^{2}=61.75 \%, \mathrm{P}=0.002\right.$ and $\mathrm{I}^{2}=60.05 \%$, $\mathrm{P}=0.007$, respectively), and 0.58 for epigastric pain $(95 \% \mathrm{CI}$, $0.34-0.97)$ by the fixed-effects model $\left(\mathrm{I}^{2}=34.00 \%, \mathrm{P}=0.157\right)$. The majority of the studies did not provide details on how they estimated the severity of adverse events.

Risk of bias in publication. Funnel plot analyses by ITT analysis revealed slight asymmetry, but Egger's test and Begg's test showed no significant asymmetry of the funnel plot (Fig. 5).

Sensitivity analysis. A sensitivity analysis was performed to interpret the reliability of the outcomes of the meta-analysis. Based on ITT analysis, the pooled RR values were established though the fixed- and random-effects models. The RRs were 1.12 (95\% CI, 1.08-1.16) and 1.12 (95\% CI, 1.06-1.19), respectively. No significant difference was found (overlapping CIs). 


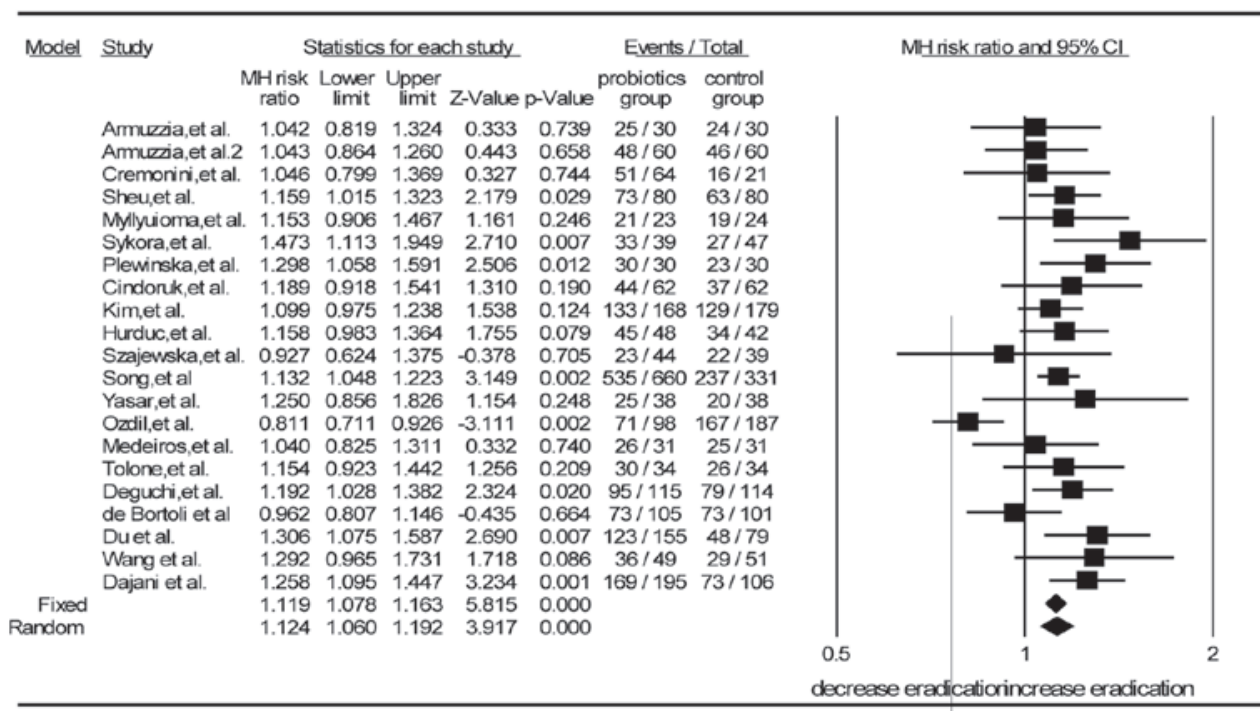

Figure 2. Forest plot comparing the eradication rate of supplementation by intention-to-treat analysis. CI, confidence interval; RR, relative risk; MH, Mantel-Haenszel.

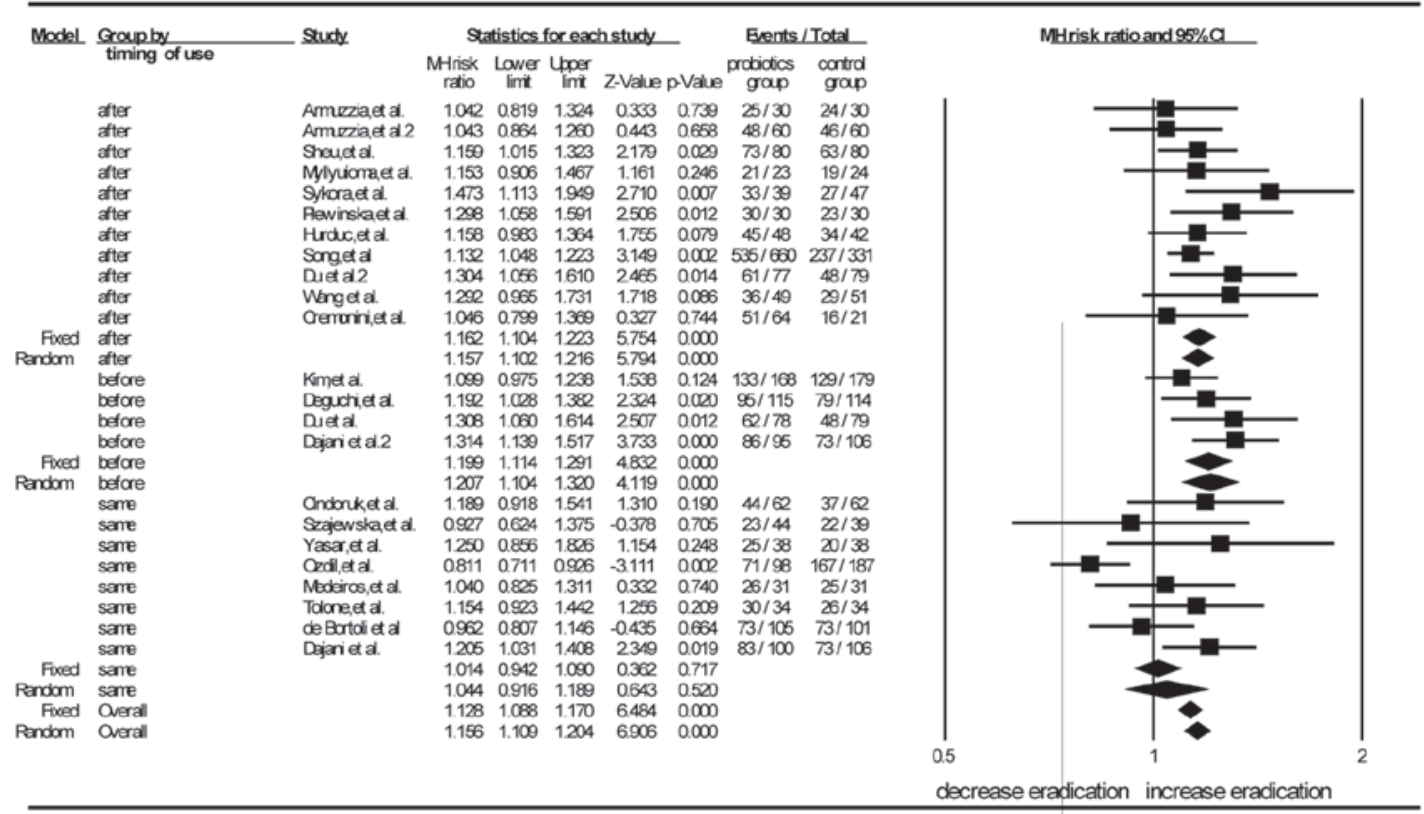

Figure 3. Forest plot of eradication rates grouped according to the timing of probiotic administration by intention-to-treat analysis. 'Before', probiotics were used prior to the eradication regimen and ended simultaneously the with regimen; 'same': probiotics were used and ended simultaneously with the eradication regimen; 'after', probiotics were used simultaneously with the eradication regimen and usage continued subsequent to the end of the regimen; CI, confidence interval; MH, Mantel-Haenszel.

When the largest study (52) was excluded from the sensitivity analysis, the $R R$ did not change significantly $(R R=1.12)$. The RRs were therefore steady.

Heterogeneity. To interpret heterogeneity in the meta-analysis, a meta-regression analysis was performed. The results showed that the timing and duration of probiotic supplementation, the duration of the eradication regimen and the quality of study were the main causes of heterogeneity.

\section{Discussion}

Probiotics, according to the World Health Organization, are defined as 'live microorganisms, which, when administered in adequate amounts, confer a health benefit on the host'. They consist of bacteria and yeasts. It has been recognized that probiotics can exhibit an inhibitory ability against $H$. pylori (5). The effects of probiotics on $H$. pylori may be due to immunologic as well as non-immunologic mechanisms: i) Competition at 


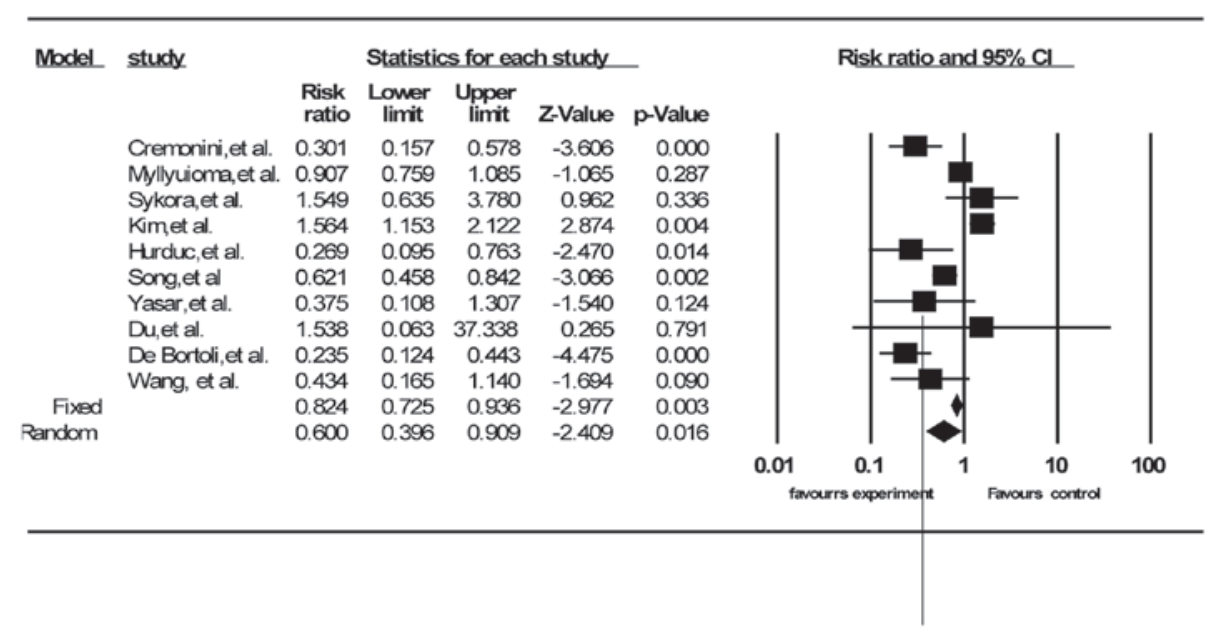

Figure 4. Effect of probiotic supplementation versus control treatment without probiotics on the incidence of total side effects. CI, confidence interval.

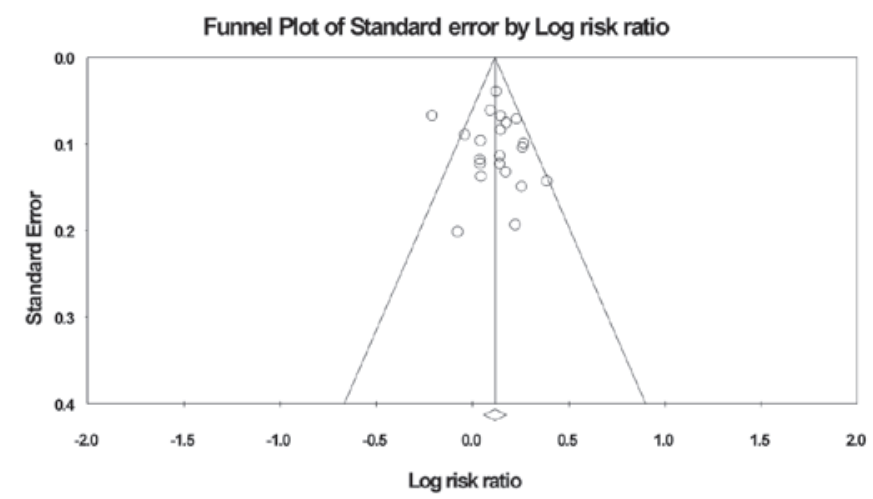

Figure 5. Funnel plot of included studies for eradication rates.

the site of the stomach mucosal epithelium (6); ii) production of substances against $H$. pylori, such as acetic, propionic or butyric acid (58); iii) regulation of immune function and secretion of immunoglobulin A to improve mucosal defensive ability (59-61); and iv) strengthening tight junctions between epithelial cells $(61,62)$.

The current results showed that probiotics could improve the eradication rate and decrease adverse events during anti-H.pylori treatment. The RRs were 1.12 (95\% CI, 1.06-1.19) and 0.62 (95\% CI, 0.40-0.91), respectively. The outcomes of the present meta-analysis were similar with several previous meta-analyses $(15,17,18)$.

The optimal timing of probiotic administration is still unknown $(23,24)$. It is generally believed that better efficacy occurs when probiotic supplementation occurs concurrently with or subsequent to antibiotic regimens. When probiotics are administered prior to antibiotic regimens, $H$. pylori is converted from a spiral to a coccoid form, which can lead to eradication failure. The timing of the addition of probiotics has been different in clinical trials $(38,56,57)$. Whether the variability affected the eradication rate of $H$. pylori is not clear. The results of the current meta-analysis suggested that probiotic supplementation could improve eradication rates when provided prior or subsequent to the standard treatment regimens, but that supplementation supplied concurrently with the regimen did not significantly improve the eradication rate. The reason behind this may be that, when probiotics and antibiotics are administered simultaneously, it is inevitable that the antibiotics restrain the growth of the probiotics, resulting in a decrease in the anti- $H$. pylori substances produced by the probiotics $(23,24)$.

An additional undetermined factor in studies to date is the appropriate duration of probiotic administration $(23,24)$. In the present meta-analysis, the duration of probiotic administration varied from 7 days to months $(38,45,47,51)$. The results suggested that a duration of $>2$ weeks could significantly improve the eradication rate for $H$. pylori infection, while a duration of $\leq 2$ weeks could not. This indicated that the long-term administration of probiotics could be beneficial during anti-H. pylori treatment; however, further investigation is required to confirm this.

Based on the subgroup analyses of probiotic species employed, it was shown that the regimens with Lactobacillus were superior to the control group regimens (RR, 1.15; 95\% CI, 1.05-1.25); however, only two RCTs used Bifidobacterium alone for adjuvant therapy during anti-H.pylori treatment. The effectiveness was slightly better than that of the control group regimens, but the difference was not statistically significant. Further investigation is therefore required to draw a definite conclusion. The use of Saccharomyces boulardii as a single supplement did not improve the eradication rate during anti-H. pylori treatment (RR, 1.05; 95\% CI, 0.89-1.23). This suggests that the administration of Saccharomyces boulardii alone may not be suitable for adjuvant treatment during anti-H. pylori therapy $(11,42,48,50,52)$.

Subgroup analyses were also performed according to different PPIs and durations of eradication regimens. It was of note that the omeprazole and rabeprazole subgroups achieved significant eradication success, while the esomeprazole, lansoprazole and pantoprazole subgroups did not, as compared with the control group. The current meta-analysis also demonstrated that the triple-therapy regimens with PPIs, amoxicillin and clarithromycin could achieve significantly higher eradication rates than the control group regimens, but the triple-therapy regimens consisting of PPIs, clarithromycin and nitroimidazole could not. 
A number of studies have indicated that the administration of probiotics can ameliorate the symptoms and reduce the adverse effects associated with eradication therapy for H. pylori, such as diarrhea, vomiting, nausea and epigastric pain $(14,35,47)$; however, certain investigations have suggested that probiotic supplementation does not reduce the incidence of side effects $(21,49)$. The side effects experienced during anti- $H$. pylori regimens were therefore examined in the current meta-analysis, which showed that the supplementation of probiotics had a substantial effect on reducing $H$. pylori therapy-related adverse reactions, particularly diarrhea and epigastric pain. The results were consistent with those of previous meta-analyses $(15,17,18)$. We believe that the application of probiotics has a beneficial effect and diminishes the discomfort during anti-H. pylori therapy.

To decrease bias in the present meta-analysis, the study selection, data extraction and assessment of study quality were performed by two reviewers. Another strength of the current meta-analysis was that it identified the majority of the RCTs published in English that used different species of probiotics as adjuvant agents for $H$. pylori treatment. The efficacy and safety of probiotics in anti-H. pylori treatment were comprehensively analyzed. The meta-regression analysis made the outcomes of the present meta-analysis reliable.

There were several limitations to the meta-analysis. Firstly, some evident heterogeneity was noted in the meta-analysis, although sub-analysis and meta-regression analysis were conducted to decrease the effects. Secondly, the language restriction could have influenced the results. There could have been a bias in the published languages, so it is likely that the present meta-analysis does not reflect all the outcomes of probiotics used for anti-H. pylori treatment. Finally, certain authors were asked for unpublished data, so the introduction of bias on that basis is possible. The Egger's and Begg's tests suggested that there could have been publication biases, and these could have affected the results of the meta-analysis.

In conclusion, the present meta-analysis showed that probiotic supplementation can improve eradication rates and reduce the adverse effects experienced during eradication therapy. In addition, probiotics appear to have enhanced effects on eradication rates when administered prior or subsequent to the standard regimens. Long-term probiotic treatment may have a superior effect to short-term probiotic administration. Lactobacillus and probiotic supplementation with multiple species appear to improve the eradication rate for $H$. pylori infection.

\section{Acknowledgements}

This study was supported by a grant from the National Science and Technology Major Projects for 'Major New Drug Innovation and Development' of China (no. 2011ZX09302-007-03). The original manuscript was edited and proofread by Medjaden Bioscience Limited. The abstract of the present study has previously been published in the proceedings for the XXVIIth International Workshop on Helicobacter and Microbiota in Chronic Digestive Inflammation and Gastric Cancer (11-13 September 2014), and in the Journal of Gastroenterology and Hepatology (vol 29, supplement S3, 2014).

\section{References}

1. Go MF: Review article: natural history and epidemiology of Helicobacter pylori infection. Aliment Pharmacol Ther 16 Suppl 1: 3-15, 2002.

2. Chey WD and Wong BC; Practice Parameters Committee of the American College of Gastroenterology: American College of Gastroenterology guideline on the management of Helicobacter pylori infection. Am J Gastroenterol 102: 1808-1825, 2007

3. Malfertheiner P, Megraud F, O'Morain CA, et al: Management of Helicobacter pylori infection - the Maastricht IV/Florence consensus report. Gut 61: 646-664, 2012.

4. Bell GD, Powell K, Burridge SM, et al: Experience with 'triple' anti-Helicobacter pylori eradication therapy: side effects and the importance of testing the pre-treatment bacterial isolate for metronidazole resistance. Aliment Pharmacol Ther 6: 427-435, 1992.

5. Bhatia SJ, Kochar N, Abraham P, Nair NG and Mehta AP: Lactobacillus acidophilus inhibits growth of Campylobacter pylori in vitro. J Clin Microbiol 27: 2328-2330, 1989.

6. Mukai T, Asasaka T, Sato E, et al: Inhibition of binding of Helicobacter pylori to the glycolipid receptors by probiotic Lactobacillus reuteri. FEMS Immunol Med Microbiol 32: 105-110, 2002.

7. Johnson-Henry KC, Mitchell DJ, Avitzur Y, et al: Probiotics reduce bacterial colonization and gastric inflammation in H. pylori-infected mice. Dig Dis Sci 49: 1095-1102, 2004.

8. Hsieh PS, Tsai YC, Chen YC, et al: Eradication of Helicobacter pylori infection by the probiotic strains Lactobacillus johnsonii MH-68 and L. salivarius ssp. salicinius AP-32. Helicobacter 17: 466-477, 2012.

9. Tursi A, Brandimarte G, Giorgetti GM and Modeo ME: Effect of Lactobacillus casei supplementation on the effectiveness and tolerability of a new second-line 10-day quadruple therapy after failure of a first attempt to cure Helicobacter pylori infection. Med Sci Monit 10: CR662-CR666, 2004.

10. Lionetti E, Indrio F, Pavone L, et al: Role of probiotics in pediatric patients with Helicobacter pylori infection: a comprehensive review of the literature. Helicobacter 15: 79-87, 2010.

11. Ozdil K, Calhan T, Sahin A, et al: Levofloxacin based sequential and triple therapy compared with standard plus probiotic combination for Helicobacter pylori eradication. Hepatogastroenterology 58: 1148-1152, 2011.

12. Yoon H, Kim N, Kim JY, et al: Effects of multistrain probiotic-containing yogurt on second-line triple therapy for Helicobacter pylori infection. J Gastroenterol Hepatol 26: 44-48, 2011.

13. Scaccianoce G, Zullo A, Hassan C, et al: Triple therapies plus different probiotics for Helicobacter pylori eradication. Eur Rev Med Pharmacol Sci 12: 251-256, 2008.

14. Ahmad K, Fatemeh F, Mehri N and Maryam S: Probiotics for the treatment of pediatric Helicobacter pylori infection: a randomized double blind clinical trial. Iran J Pediatr 23: 79-84, 2013.

15. Tong JL, Ran ZH, Shen J, Zhang CX and Xiao SD: Meta-analysis: the effect of supplementation with probiotics on eradication rates and adverse events during Helicobacter pylori eradication therapy. Aliment Pharmacol Ther 25: 155-168, 2007.

16. Szajewska H, Horvath A and Piwowarczyk A: Meta-analysis: the effects of Saccharomyces boulardii supplementation on Helicobacter pylori eradication rates and side effects during treatment. Aliment Pharmacol Ther 32: 1069-1079, 2010.

17. Zou J, Dong J and Yu X: Meta-analysis: Lactobacillus containing quadruple therapy versus standard triple first-line therapy for Helicobacter pylori eradication. Helicobacter 14: 97-107, 2009.

18. Wang ZH, Gao QY and Fang JY: Meta-analysis of the efficacy and safety of Lactobacillus-containing and Bifidobacterium-containing probiotic compound preparation in Helicobacter pylori eradication therapy. J Clin Gastroenterol 47: 25-32, 2013.

19. Sachdeva A and Nagpal J: Effect of fermented milk-based probiotic preparations on Helicobacter pylori eradication: a systematic review and meta-analysis of randomized-controlled trials. Eur J Gastroenterol Hepatol 21: 45-53, 2009.

20. Zheng X, Lyu L and Mei Z: Lactobacillus-containing probiotic supplementation increases Helicobacter pylori eradication rate: evidence from a meta-analysis. Rev Esp Enferm Dig 105: 445-453, 2013. 
21. Shavakhi A, Tabesh E, Yaghoutkar A, et al: The effects of multistrain probiotic compound on bismuth-containing quadruple therapy for Helicobacter pylori infection: a randomized placebo-controlled triple-blind study. Helicobacter 18: 280-284, 2013.

22. Navarro-Rodriguez T, Silva FM, Barbuti RC, et al: Association of a probiotic to a Helicobacter pylori eradication regimen does not increase efficacy or decreases the adverse effects of the treatment a prospective, randomized, double-blind, placebo-controlled study. BMC Gastroenterol 13: 56, 2013.

23. Matsushima M and Takagi A: 'Is it effective?' to 'How to use it?' the era has changed in probiotics and functional food products against Helicobacter pylori infection. J Gastroenterol Hepatol 27: 851-853, 2012.

24. Boyanova L and Mitov I: Coadministration of probiotics with antibiotics: why, when and for how long? Expert Rev Anti Infect Ther 10: 407-409, 2012.

25. Liberati A, Altman DG, Tetzlaff J, et al: The PRISMA statement for reporting systematic reviews and meta-analyses of studies that evaluate healthcare interventions: explanation and elaboration. BMJ 339: b2700, 2009.

26. Jadad AR, Moore RA, Carroll D, et al: Assessing the quality of reports of randomized clinical trials: is blinding necessary? Control Clin Trials 17: 1-12, 1996.

27. Downs SH and Black N: The feasibility of creating a checklist for the assessment of the methodological quality both of randomised and non-randomised studies of health care interventions. J Epidemiol Community Health 52: 377-384, 1998.

28. Sahagún-Flores JE, López-Peña LS, de la Cruz-Ramírez Jaimes J, et al: Eradication of Helicobacter pylori: triple treatment scheme plus Lactobacillus vs. triple treatment alone. Cir Cir 75: 333-336, 2007 (In Spanish)

29. Ziemniak W: Efficacy of Helicobacter pylori eradication taking into account its resistance to antibiotics. J Physiol Pharmacol 57 Suppl 3: 123-141, 2006.

30. Sheu BS, Cheng HC, Kao AW, et al: Pretreatment with Lactobacillus- and Bifidobacterium-containing yogurt can improve the efficacy of quadruple therapy in eradicating residual Helicobacter pylori infection after failed triple therapy. Am J Clin Nutr 83: 864-869, 2006

31. Lionetti E, Miniello VL,Castellaneta SP, et al: Lactobacillus reuteri therapy to reduce side-effects during anti-Helicobacter pylori treatment in children: a randomized placebo controlled trial. Aliment Pharmacol Ther 24: 1461-1468, 2006.

32. Manfredi M, Bizzarri B, Sacchero RI, et al: Helicobacter pylori infection in clinical practice: probiotics and a combination of probiotics + lactoferrin improve compliance, but not eradication, in sequential therapy. Helicobacter 17: 254-263, 2012

33. Xu C, Xiao L and Zou H: Effect of birid triple viable on peptic ulcer patients with Helicobacter pylori infection. Zhong Nan Da Xue Xue Bao Yi Xue Ban 35: 1000-1004, 2010 (In Chinese)

34. Park SK, Park DI, Choi JS, et al: The effect of probiotics on Helicobacter pylori eradication. Hepatogastroenterology 54 2032-2036, 2007.

35. Bekar O, Yilmaz Y and Gulten M: Kefir improves the efficacy and tolerability of triple therapy in eradicating Helicobacter pylori. J Med Food 14: 344-347, 2011.

36. Nista EC, Candelli M, Cremonini F, et al: Bacillus clausii therapy to reduce side-effects of anti-Helicobacter pylori treatment: randomized, double-blind, placebo controlled trial. Aliment Pharmacol Ther 20: 1181-1188, 2004.

37. Canducci F, Armuzzi A, Cremonini F, et al: A lyophilized and inactivated culture of Lactobacillus acidophilus increases Helicobacter pylori eradication rates. Aliment Pharmacol Ther 14 $1625-1629,2000$

38. Wang YH and Huang Y: Effect of Lactobacillus acidophilus and Bifidobacterium bifidum supplementation to standard triple therapy on Helicobacter pylori eradication and dynamic changes in intestinal flora. World J Microbiol Biotechnol 30: 847-853, 2014.

39. Dajani AI, Abu Hammour AM, Yang DH, et al: Do probiotics improve eradication response to Helicobacter pylori on standard triple or sequential therapy? Saudi J Gastroenterol 19: 113-120, 2013

40. Armuzzi A, Cremonini F, Bartolozzi F, et al: The effect of ora administration of Lactobacillus GG on antibiotic-associated gastrointestinal side-effects during Helicobacter pylori eradication therapy. Aliment Pharmacol Ther 15: 163-169, 2001.

41. Armuzzi A,Cremonini F, Ojetti V,etal: Effect of Lactobacillus GG supplementation on antibiotic-associated gastrointestinal side effects during Helicobacter pylori eradication therapy: a pilot study. Digestion 63: 1-7, 2001
42. Cremonini F, Di Caro S, Covino M, et al: Effect of different probiotic preparations on anti-Helicobacter pylori therapy-related side effects: a parallel group, triple blind, placebo-controlled study. Am J Gastroenterol 97: 2744-2749, 2002.

43. Sheu BS, Wu JJ, Lo CY, et al: Impact of supplement with Lactobacillus- and Bifidobacterium-containing yogurt on triple therapy for Helicobacter pylori eradication. Aliment Pharmacol Ther 16: 1669-1675, 2002

44. Myllyluoma E, Veijola L, Ahlroos T, et al: Probiotic supplementation improves tolerance to Helicobacter pylori eradication therapy - a placebo-controlled, double-blind randomized pilot study. Aliment Pharmacol Ther 21: 1263-1272, 2005.

45. Sýkora J, Valecková K, Amlerová J, et al: Effects of a specially designed fermented milk product containing probiotic Lactobacillus casei DN-114 001 and the eradication of $H$. pylori in children: a prospective randomized double-blind study. J Clin Gastroenterol 39: 692-698, 2005.

46. Plewińska EM, Płaneta-Małecka I, Bak-Romaniszyn L, Czkwianianc E and Małecka-Panas E: Probiotics in the treatment of Helicobacter pylori infection in children. Gastroenterologia Polska 13: 315-319, 2006.

47. de Bortoli N, Leonardi G, Ciancia E, et al: Helicobacter pylori eradication: a randomized prospective study of triple therapy versus triple therapy plus lactoferrin and probiotics. Am J Gastroenterol 102: 951-956, 2007.

48. Cindoruk M,Erkan G,Karakan T,Dursun A and Unal S: Efficacy and safety of Saccharomyces boulardii in the 14-day triple anti-Helicobacter pylori therapy: a prospective randomized placebo-controlled double-blind study. Helicobacter 12: 309-316, 2007.

49. Kim MN, Kim N, Lee SH, et al: The effects of probiotics on PPI-triple therapy for Helicobacter pylori eradication. Helicobacter 13: 261-268, 2008.

50. Hurduc V, Plesca D, Dragomir D, Sajin M and Vandenplas Y: A randomized, open trial evaluating the effect of Saccharomyces boulardii on the eradication rate of Helicobacter pylori infection in children. Acta Paediatr 98: 127-131, 2009.

51. Szajewska H, Albrecht P and Topczewska-Cabanek A: Randomized, double-blind, placebo-controlled trial: effect of Lactobacillus GG supplementation on Helicobacter pylori eradication rates and side effects during treatment in children. J Pediatr Gastroenterol Nutr 48: 431-436, 2009.

52. Song MJ, Park DI, Park JH, et al: The effect of probiotics and mucoprotective agents on PPI-based triple therapy for eradication of Helicobacter pylori. Helicobacter 15: 206-213, 2010.

53. Yasar B, Abut E, Kayadibı H, et al: Efficacy of probiotics in Helicobacter pylori eradication therapy. Turk J Gastroenterol 21: 212-217, 2010

54. Medeiros JA, Gonçalves TM, Boyanova L, et al: Evaluation of Helicobacter pylori eradication by triple therapy plus Lactobacillus acidophilus compared to triple therapy alone. Eur J Clin Microbiol Infect Dis 30: 555-559, 2011.

55. Deguchi R, Nakaminami H, Rimbara E, et al: Effect of pretreatment with Lactobacillus gasseri OLL2716 on first-line Helicobacter pylori eradication therapy. J Gastroenterol Hepatol 27: 888-892, 2012.

56. Du YQ, Su T, Fan JG, et al: Adjuvant probiotics improve the eradication effect of triple therapy for Helicobacter pylori infection. World J Gastroenterol 18: 6302-6307, 2012.

57. Tolone S, Pellino V, Vitaliti G, Lanzafame A and Tolone C: Evaluation of Helicobacter pylori eradication in pediatric patients by triple therapy plus lactoferrin and probiotics compared to triple therapy alone. Ital J Pediatr 38: 63, 2012.

58. Gotteland M, Brunser O and Cruchet S: Systematic review: are probiotics useful in controlling gastric colonization by Helicobacter pylori? Aliment Pharmacol Ther 23: 1077-1086, 2006.

59. Yang YJ and Sheu BS: Probiotics-containing yogurts suppress Helicobacter pylori load and modify immune response and intestinal microbiota in the Helicobacter pylori-infected children. Helicobacter 17: 297-304, 2012.

60. Kwon HK, Kim GC, Kim Y, et al: Amelioration of experimental autoimmune encephalomyelitis by probiotic mixture is mediated by a shift in T helper cell immune response. Clin Immunol 146: 217-227, 2013

61. Sultana R, McBain AJ and O'Neill CA: Strain-dependent augmentation of tight-junction barrier function in human primary epidermal keratinocytes by Lactobacillus and Bifidobacterium lysates. Appl Environ Microbiol 79: 4887-4894, 2013.

62. Yeung CY, Chiang Chiau JS, Chan WT, et al: In vitro prevention of Salmonella lipopolysaccharide-induced damages in epithelial barrier function by various Lactobacillus strains. Gastroenterol Res Pract 2013: 973209, 2013. 